\title{
Efecto de la sustitución de grasa dorsal de cerdo por aceite de aguacate en la calidad de salchichas de pollo tipo suiza
}

\section{(Effect of replacing pork backfat with avocado oil on the quality of chicken sausages "Swiss type")}

\author{
Adriàn Moreno Vaca ${ }^{1}$, Priscila Maldonado Pacheco ${ }^{2}$
}

\begin{abstract}
Resumen:
Se desarrolló un embutido con aceite de aguacate en sustitución total de la grasa dorsal de cerdo contenida en su formulación. El análisis de perfil lipídico para la grasa dorsal de cerdo reportó: AGS 35.45\%; AGMI 41.95\%; AGPI 20.71\%. Para el aceite de aguacate: AGS 18.66\%; AGMI 68.37\%; AGPI 12.19\%. Los tratamientos utilizados correspondientes a los porcentajes de contenido de aceite de aguacate como sustituto de la grasa dorsal de cerdo en su formulación fueron: $T_{1}=50 \%, T_{2}=75 \%$ y $T_{3}=100 \%$. Se determinó la aceptabilidad sensorial de los tratamientos. No existieron diferencias estadísticamente significativas. Se escogió el tratamiento con más alta puntuación como el mejor, tratamiento $T_{3}$. Se realizó la caracterización físico-química y microbiológica del tratamiento $\mathrm{T}_{3}$ y de un tratamiento el cual no tuvo en su formulación aceite de aguacate, tratamiento $\mathrm{T}_{0}$. Proteína en $\mathrm{T}_{0}$ fue $13.23 \%$ y en $\mathrm{T}_{3} 12.56 \%$. Grasa total, AGS y AGPI en $\mathrm{T}_{3}$ se redujeron en $3.7 \%, 5.24 \%$ y $2.53 \%$ respectivamente. AGMI presentó un incremento de $3.51 \%$ en $\mathrm{T}_{3}$. Ácidos trans fue $0 \%$ para ambos tratamientos. Los resultados obtenidos en la caracterización microbiológica se encontraron dentro de los requerimientos establecidos en la NTE INEN 1338:12.
\end{abstract}

Palabras clave: Sustitución; salchicha; grasa; aceite; aguacate

\begin{abstract}
:
A kind of chicken sausage "Swiss type" was developed with avocado oil content to replace all fat from pork back fat in its formulation. Analysis of lipid profile for pork back fat reported: SFA $35.45 \%$; MUFA $41.95 \%$; PUFA $20.71 \%$. For avocado oil: SFA $18.66 \%$; MUFA $68.37 \%$; PUFA $12.19 \%$. Treatments used which corresponding to the percentages of avocado oil content as a substitute for pork back fat in their formulation were: $\mathrm{T}_{1}=50 \%, \mathrm{~T}_{2}=75 \%, \mathrm{~T}_{3}=100 \%$. Sensory acceptability of treatments was performed. There were no statistically significant differences. Treatment $T_{3}$ with highest score was chosen as the best treatment. Physicochemical and microbiological characterization was performed for $\mathrm{T}_{3}$ treatment and for a treatment which didn't have in its formulation avocado oil, $\mathrm{T}_{0}$ treatment. $\mathrm{T}_{0}$. Protein was $13.23 \%$ in $\mathrm{T}_{0}$ and $12.56 \%$ in $\mathrm{T}_{3}$. Total fat, SFA and PUFA in $\mathrm{T}_{3}$ were reduced in $3.7 \%, 5.24 \%$ and $2.53 \%$ respectively. MUFA showed an increase of $3.51 \%$ in $\mathrm{T}_{3}$. Trans acids was $0 \%$ for both treatments. The results obtained were located within the acceptable range established in the NTE (INEN) 1338:12.
\end{abstract}

Keywords: substitution; sausage; fat; oil; avocado.

\footnotetext{
${ }^{1}$ Universidad Tecnológica Equinoccial, Quito - Ecuador (mvra34136@ute.edu.ec)

${ }^{2}$ Universidad Tecnológica Equinoccial, Quito - Ecuador (bmaldonado@ute.edu.ec)
} 


\section{Introducción}

El consumo de grasas saturadas en exceso constituye un factor de riesgo para la salud humana favoreciendo el desarrollo de patologías como obesidad, enfermedades cardiovasculares, hipertensión, diabetes y dislipidemias, las cuales se encuentran entre las principales causas de mortalidad en el país (INEC, 2011).

El Ministerio de Salud Pública del Ecuador a través de un comunicado de prensa dio a conocer que $82 \%$ de alimentos procesados ofertados en Ecuador contienen grasa, sal y azúcar en niveles altos y por el contrario solamente un $2 \%$ tienen niveles bajos. (LA HORA, 2013. 22 de noviembre),

Actualmente, en la elaboración de salchicha, existe una tendencia dirigida a la disminución del contenido de grasa saturada del producto por razones de salud, así como la incorporación de grasa vegetal en la formulación (García, Palo \& Salas 2008).

En Turquía, en la Universidad de Ege se desarrollaron embutidos en los cuales se reemplazó parcialmente la grasa animal por aceite de avellana de 60 al $90 \%$, conservando la estabilidad en el embutido (Yildiz \& Serdaroglu 2012). El estudio expone que el reemplazo con aceite de avellana mejoró la calidad nutricional, bajando el colesterol e incrementando el contenido de ácidos grasos monoinsaturados y poliinsaturados sin que esto provocara un efecto adverso en la emulsificación o en los atributos sensoriales.

Si bien existen trabajos asociados al reemplazo de grasa dorsal de cerdo por aceite vegetal, no se ha realizado un estudio del efecto en la calidad de salchichas de pollo tipo suiza utilizando aceite de aguacate como sustituto parcial de la grasa dorsal de cerdo en la obtención de este tipo de producto.

El objetivo del presente trabajo fue estudiar el efecto de la sustitución de la grasa dorsal de cerdo por aceite de aguacate en la calidad de salchichas de pollo tipo suiza.

\section{Metodología}

\subsection{Elaboración de salchicha de pollo tipo suiza}

La carne de pollo, grasa dorsal de cerdo, aceite de aguacate y tripa sintética se obtuvo de proveedores locales. Se retiró las venas, exceso de grasa, tendones, huesos y otras partes de la carne de pollo que no se utilizan en el proceso. Se realizó adicionalmente un análisis de $\mathrm{pH}$ de la carne de pollo con el fin de comprobar su aptitud para la elaboración del embutido. Se congeló la materia prima a temperatura de $-8^{\circ} \mathrm{C}$ antes del proceso de picado. Se procedió a cortar la grasa en porciones de 2 a $3 \mathrm{~cm}$ y la carne en porciones de 5 a 10 
cm. La carne y la grasa se molieron utilizando un molino industrial HOBBART modelo 4B12. Para la carne de pollo se utilizó el disco de $5 \mathrm{~mm}$ y para la grasa animal se utiliza el disco de $9 \mathrm{~mm}$. Se colocó la carne en el en el cutter marca TALSA modelo T-3394 hasta obtener un granulo fino, con una temperatura máxima de entre 10 - $12{ }^{\circ} \mathrm{C}$. Se adicionó sal, nitritos, fosfatos y gradualmente la tercera parte del hielo previamente picado en un triturador de hielo, se añadió el hielo en función de la temperatura que mostró el cutter y al observar que se inició la solubilización de las proteínas con un incremento incontrolable de la temperatura se añadió gradualmente el hielo restante de la formulación. Posteriormente se adicionó el conservante INBAC junto con las especias. Se agregó el aceite de aguacate en diferentes porcentajes de sustitución de la grasa animal: $\mathrm{T} 0=0 \%$ de aceite $\mathrm{T} 1=50 \%$ de aceite, T2= $75 \%$ de aceite, T3=100\% de aceite. En todos los casos el porcentaje restante fue grasa dorsal de cerdo. Se obtuvo la emulsión cárnica resultante del cutter y se procedió a dejarla en reposo por 1 hora a una temperatura de $5^{\circ} \mathrm{C}$. Se procedió a colocar la emulsión en la embutidora manual marca SIRMAN serie 03LOO898, seguidamente se embutió en tripas artificiales de calibre 18. El producto que ha sido embutido se llevó a escaldado en agua; se inició con una temperatura de $40{ }^{\circ} \mathrm{C}$ para posteriormente controlar que se mantenga entre los 76 - $80^{\circ} \mathrm{C}$. El escaldado se realizó por aproximadamente 60 minutos hasta que la temperatura interna del producto llegó a los 68 - $72 \stackrel{\circ}{\circ}$. Una vez alcanzada la temperatura interna requerida, se sometió a los embutidos a un choque térmico en agua hasta que la temperatura interna del embutido alcanzó $22{ }^{\circ} \mathrm{C}$. Se eliminó el exceso de agua de la superficie de los embutidos para ser empacados al vacío. Las salchichas empacadas se llevaron a refrigeración a una temperatura recomendada de 0-4 ํㅡ por 24 horas.

\subsection{Análisis de aceptabilidad sensorial}

Se evaluó la aceptabilidad sensorial de los atributos de color, olor, sabor, textura al masticar (homogeneidad y dureza). Se elaboró un test mediante escalas hedónicas con preguntas acerca de los atributos color, olor, sabor y textura al masticar; se utilizó una escala semi estructurada de nueve puntos, donde 9 fue la máxima puntuación de aceptabilidad. Cada muestra se identificó con un código de 3 dígitos obtenido de una tabla de números aleatorios. Se eligió un panel de evaluadores los cuales analizaron los productos elaborados con aceite de aguacate en su formulación y evaluaron según el test proporcionado.

\subsection{Análisis fisico-químicos}

Se efectuaron determinaciones de proteína, método AOAC 928,08 para clasificar al embutido dentro de norma INEN; grasa total, método AOAC 920,39 con la finalidad de dar cumplimiento a la legislación de etiquetado nutricioal; perfil lipídico y grasas trans, método AOAC 991,39, para establecer las diferencias en el perfil lipídico de la salchicha elaborada con grasa dorsal y con aceite de aguacate. 


\subsection{Análisis microbiológicos}

Se realizaron conteos totales de Escherichia coli, método AOAC 991, 14; Aerobios mesófilos, método AOAC 990,12; Staphylococcus aureus, método AOAC 2003,07; Salmonella, método AOAC 967 25.26.27.

Se utilizaron placas petrifilm $3 \mathrm{M}^{\mathrm{TM}}$ correspondientes a cada microorganismo a identificar. En el proceso de inoculación de la muestra en los análisis microbiológicos se utilizó una micropipeta 10-1000 $\mu \mathrm{L}$ marca GLASSCO.

Estos análisis son un requisito solicitado en la Norma INEN 1338 - 2012.

\subsection{Análisis de datos}

En el análisis de aceptabilidad sensorial se empleó un diseño de bloques completos al azar (DBCA). Para los análisis físico-químicos se empleó un diseño completamente al azar (DCA). Los resultados en ambos casos fueron procesados mediante un análisis de varianza (ANOVA) y las medias comparadas con una significancia de 0,05 usando el software estadístico STATGRAPHICS CENTURION XVI.

\section{Resultados y Discusión}

\subsection{Determinación de perfil lipídico de grasa dorsal de cerdo}

La determinación del perfil lipídico se realizó mediante cromatografía de gases. En la Tabla 1 se muestra los valores del perfil lipídico obtenidos de la grasa dorsal de cerdo. Se reportó un contenido de $35.45 \%$ de AGS, proveniente de los ácidos grasos mirístico, palmítico y esteárico; $41.95 \%$ de AGMI proveniente de los ácidos grasos palmitoleico y oleico; $20.71 \%$ de AGPI proveniente de los ácidos linoleico y linolénico

Tabla 1. Perfil Lipídico de grasa dorsal de cerdo

\begin{tabular}{llr}
\hline Ácido Graso & $\begin{array}{l}\text { Número } \\
\text { lipídico }\end{array}$ & \multirow{2}{*}{$\%$} \\
\hline Mirístico & $\mathrm{C} 14: 0$ & 1.45 \\
Palmítico & $\mathrm{C} 16: 0$ & 23.09 \\
Palmitoleico & $\mathrm{C} 16: 1$ & 1.71 \\
Esteárico & $\mathrm{C} 18: 0$ & 10.91 \\
Oleico & $\mathrm{C} 18: 1$ & 40.24 \\
Linoleico & $\mathrm{C} 18: 2$ & 20.11 \\
Linolénico & $\mathrm{C} 18: 3$ & 0.60 \\
\hline Ácidos Grasos Saturados & & 35.45 \\
Ácidos Grasos Monoinsaturados & & 41.95 \\
Ácidos Grasos Poliinsaturados & & 20.71 \\
\hline
\end{tabular}

${ }^{*}$ Cada valor es el promedio de los resultados obtenidos. 
Los resultados obtenidos son similares a los reportados por Bañon et al. (2000) para el perfil lipídico de grasa dorsal de cerdo, quien describe que estos valores refieren a una grasa de buena calidad, sin embargo la grasa utilizada en el presente estudio puede considerarse como una grasa blanda, ya que el porcentaje de ácido linoleico es mayor al $15 \%$.

\subsection{Determinación de perfil lipídico de aceite de aguacate}

En la Tabla 2 se muestra los valores del perfil lipídico obtenidos del aceite de aguacate. Se reportó un contenido de $18.66 \%$ de AGS, proveniente de los ácidos grasos palmítico y esteárico; $68.37 \%$ de AGMI proveniente de los ácidos grasos palmitoleico y oleico; $12.19 \%$ de AGPI proveniente de los ácidos linolénico y linoleico.

Tabla 2: Perfil lipídico de aceite de aguacate

\begin{tabular}{llr}
\hline Ácido Graso & $\begin{array}{l}\text { Número } \\
\text { lipídico }\end{array}$ & \multicolumn{1}{c}{$\%$} \\
\hline Palmítico & $\mathrm{C} 16: 0$ & 18.00 \\
Palmitoleico & $\mathrm{C} 16: 1$ & 9.42 \\
Esteárico & $\mathrm{C} 18: 0$ & 0.61 \\
Oleico & $\mathrm{C} 18: 1$ & 58.95 \\
Linoleico & $\mathrm{C} 18: 2$ & 11.50 \\
Linolénico & $\mathrm{C} 18: 3$ & 0.69 \\
\hline Ácidos Grasos Saturados & & 18.61 \\
Ácidos Grasos Monoinsaturados & & 68.37 \\
Ácidos Grasos Poliinsaturados & & 12.19 \\
\hline
\end{tabular}

*Cada valor es el promedio de los resultados obtenidos.

Los resultados obtenidos para el perfil lipídico del aceite de aguacate están de acuerdo a los valores presentados por (Acosta 2011) que indican $16.99 \%$ de AGS, $69.89 \%$ de AGMI, $13.09 \%$ de AGPI.

\subsection{Determinación de aceptabilidad sensorial}

Los resultados de la evaluación sensorial de los tratamientos $T_{1}, T_{2}$ y $T_{3}$ se describen a continuación en la Tabla 3, en la cual se presenta las medias y desviación estándar de cada atributo evaluado.

Tabla 3. Resumen de medias por atributos

\begin{tabular}{ccccc}
\hline \multicolumn{5}{c}{ Atributos $^{2}$} \\
\hline Tratamientos $^{1}$ & Color & Olor & Sabor & Textura al masticar \\
$\mathrm{T}_{1}$ & $5.00 \pm 2.63^{\mathrm{a}}$ & $5.67 \pm 2.16^{\mathrm{a}}$ & $5.93 \pm 2.47^{\mathrm{a}}$ & $6.22 \pm 2.39^{\mathrm{a}}$ \\
$\mathrm{T}_{2}$ & $5.13 \pm 2.72^{\mathrm{a}}$ & $5.61 \pm 2.03^{\mathrm{a}}$ & $5.32 \pm 2.50^{\mathrm{a}}$ & $5.68 \pm 2.58^{\mathrm{a}}$ \\
$\mathrm{T}_{3}$ & $5.86 \pm 2.59^{\mathrm{a}}$ & $6.04 \pm 2.29^{\mathrm{a}}$ & $5.52 \pm 2.78^{\mathrm{a}}$ & $5.88 \pm 2.50^{\mathrm{a}}$ \\
\hline
\end{tabular}

\footnotetext{
${ }^{1}$ Tratamientos con contenido de aceite de aguacate en sustitución de la grasa animal: T1=50 $\%$ de aceite, T2= $75 \%$ de aceite, T3=100\% de aceite.

${ }^{2}$ Letras diferentes en una misma columna indica diferencia significativa $(P<0.05)$.

Cada valor es el promedio \pm la Desviación Standard de los resultados obtenidos. $\mathbf{n}=100$
} 


\subsubsection{Olor}

Los promedios de los resultados obtenidos para el atributo olor agrupados por tratamientos se muestran en la Figura 1.

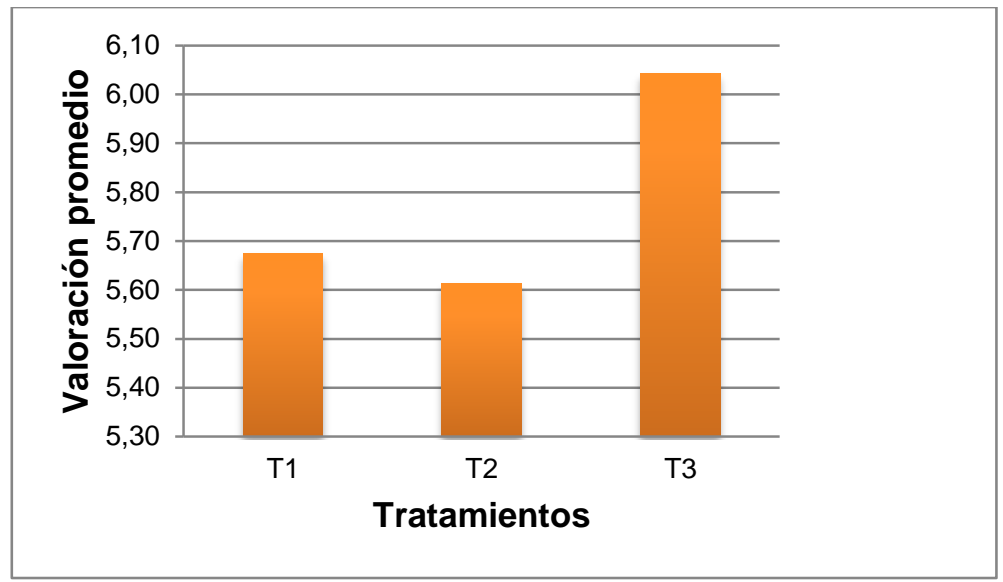

Figura 1. Calificación promedio obtenida para el atributo olor en los tratamientos $T_{1}, T_{2}, T_{3}$

En el atributo olor la muestra con el $100 \%$ de grasa dorsal presenta la mayor aceptación. (Banda 2010), reportan resultados similares, ya que en la evaluación estadística de los datos de aceptabilidad del atributo olor, no se encontraron diferencias importantes, sin embargo se observó que el tratamiento con mejor aceptabilidad era aquel en el que se utilizaba el porcentaje más alto de grasa vegetal en sustitución de la grasa dorsal de cerdo.

El mejoramiento de la percepción en el aroma del embutido al utilizar aceite de aguacate puede deberse a que el aguacate contiene 25 compuestos de aroma conocidos como "volátiles de aroma", entre los cuales se encuentra el pentanal; este compuesto parecería ser responsable del olor y un ligero sabor a nueces del aguacate maduro Haas (Obenland 2012). La variedad de aguacate Haas es utilizada en la elaboración del aceite usado para la presente investigación. En el proceso de obtención del aceite de aguacate se logra mantener todas las propiedades originales de la fruta (Olaeta 2003).

\subsubsection{Color}

Los promedios de los resultados obtenidos para el atributo color agrupados por tratamientos se muestran en la Figura 2.

Como se evidencia en la gráfica al evaluar el atributo de color la formulación con el $100 \%$ de aceite de aguacate presenta mayor aceptación por el consumidor. Pascual et al. (2002), reportan resultados similares al evaluar el atributo color, señalan que en las pruebas estadísticas realizadas no existían diferencias significativas entre las formulaciones donde se utilizaba aceite de oliva en sustitución parcial de la grasa dorsal de cerdo en salchichas tipo Frankfurt. 


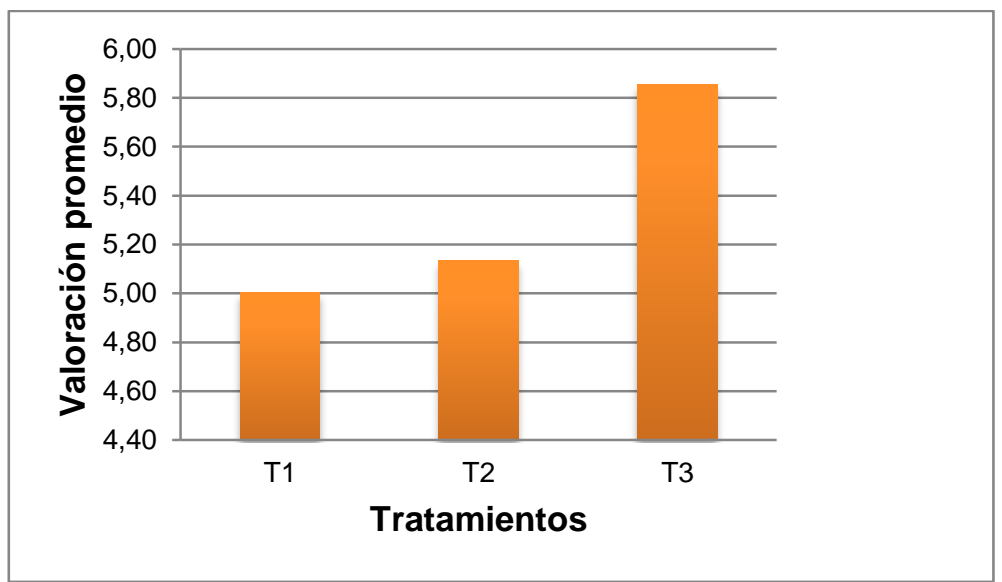

Figura 2. Calificación promedio obtenida para el atributo color en los tratamientos $T_{1}, T_{2}, T_{3}$

(Banda 2010), señala que no se encontraron diferencias significativas para el atributo color y se estableció que el mejor tratamiento fue aquel que contenía el porcentaje más alto de grasa vegetal en sustitución de la grasa de cerdo, que en ese caso fue $75 \%$.

\subsubsection{Sabor}

Los promedios de los resultados obtenidos para el atributo sabor agrupados por tratamientos se muestran en la Figura 3.

A diferencia de los resultados de olor y color en el atributo de sabor la muestra con menor contenido de aceite de aguacate presenta la mayor aceptación. En el estudio realizado por Pascual et al. (2002), reportan de igual manera que no se encontró diferencias significativas para el atributo sabor entre los ensayos realizados, adicionalmente señalan que en trabajos similares en los que se utilizó aceite de oliva, girasol, maíz y soya tampoco se encontraron diferencias significativas respecto al sabor.

Selgas et al. (2005), indica que la grasa animal contribuye al sabor de los productos cárnicos, logrando de esta manera mejorar la percepción de los consumidores hacia los mismos.

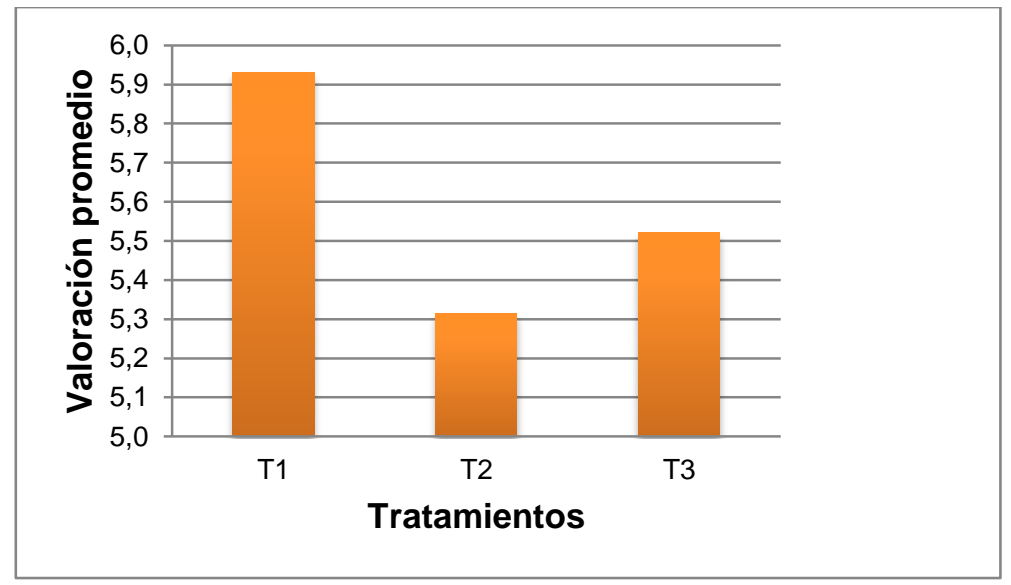

Figura 3. Calificación promedio obtenida para el atributo sabor en los tratamientos $T_{1}, T_{2}, T_{3}$ 


\subsubsection{Textura}

Los promedios de los resultados obtenidos para el atributo textura agrupados por tratamientos se muestran en la Figura 4.

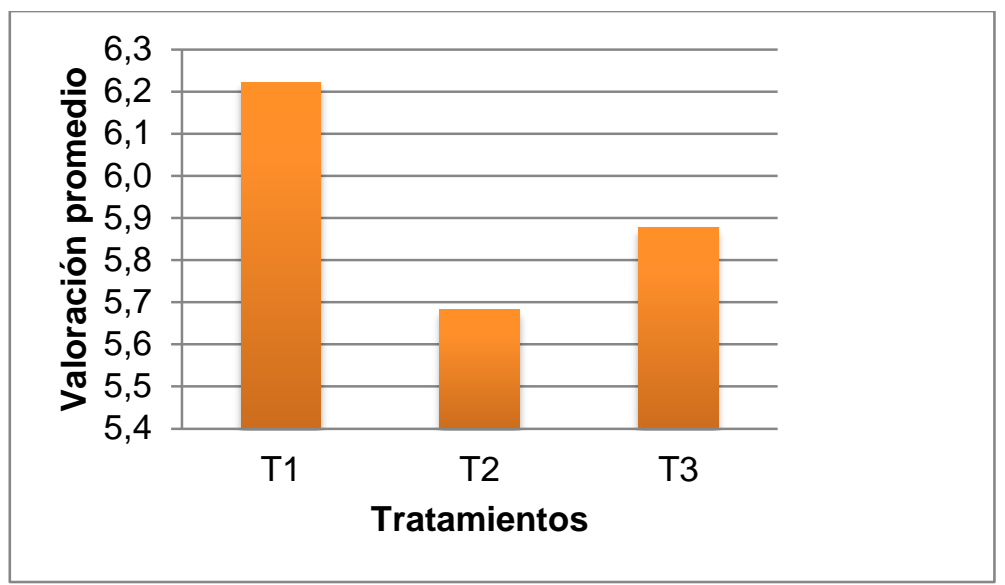

Figura 4. Calificación promedio obtenida para el atributo textura en los tratamientos $T_{1}, T_{2}, T_{3}$

Al igual que en sabor la muestra con menor porcentaje de aceite es la que presenta mayor preferencia por el consumidor.

Pascual et al. (2002), reporta resultados de no haber encontrado diferencias importantes en la evaluación sensorial para el atributo textura, sin embargo explica que se encontró una mayor preferencia para el tratamiento en el cual utilizó un menor porcentaje de aceite de oliva en sustitución parcial de la grasa de cerdo en salchichas Frankfurt. Adicionalmente se señala que el empleo de grasa animal logra ablandar el producto incrementando de esta manera la terneza.

\subsection{Selección del mejor tratamiento}

El resumen de resultados obtenidos en la evaluación de aceptabilidad sensorial para cada tratamiento se muestran en la Tabla 4.

Tabla Error! No text of specified style in document.. Resumen de medias por tratamientos

\begin{tabular}{cc}
\hline Tratamientos $^{1}$ & Media $^{2}$ \\
\hline $\mathrm{T}_{1}$ & $5.71 \pm 0.52^{\mathrm{a}}$ \\
$\mathrm{T}_{2}$ & $5.44 \pm 0.26^{\mathrm{a}}$ \\
$\mathrm{T}_{3}$ & $5.82 \pm 0.22^{\mathrm{a}}$ \\
\hline
\end{tabular}

${ }^{1}$ Porcentajes de contenido de aceite de aguacate en sustitución de la grasa animal: $\mathrm{T} 1=$ $50 \%$ de aceite, $\mathrm{T} 2=75 \%$ de aceite, $\mathrm{T} 3=100 \%$ de aceite.

${ }^{2}$ Letras diferentes en una misma columna indica diferencia significativa $(P<0.05) . \mathbf{n}=100$

No se encontraron diferencias significativas entre cada tratamiento, por lo cual el tratamiento que presentó mejores características de aceptabilidad de acuerdo a su media fue el 
tratamiento T3 que contenía $100 \%$ de aceite de aguacate en sustitución de la grasa dorsal de cerdo.

En la Figura 6 se aprecia la calificación promedio de los atributos evaluados por cada tratamiento.

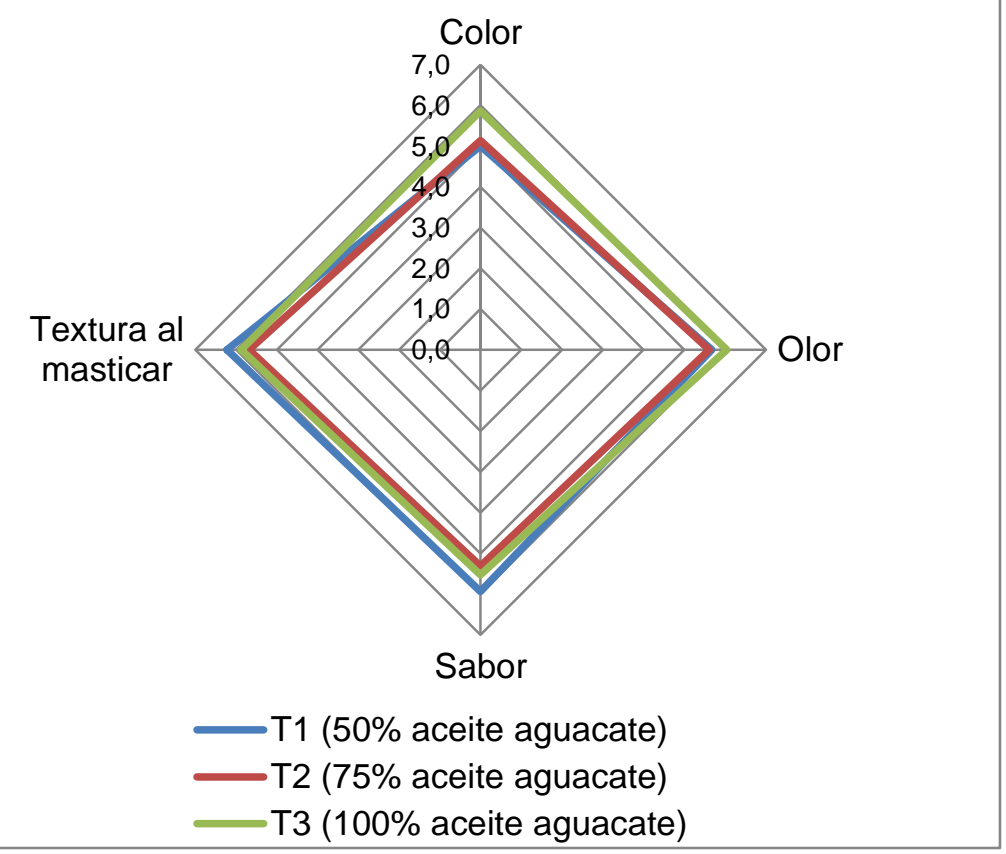

Figura 1. Perfil descriptivo de los tratamientos en función a su contenido de aceite

García et al. (2008), reportan resultados similares, ya que al no encontrar diferencias significativas para las pruebas sensoriales de color, olor y textura se eligió la muestra con el mejor puntaje promedio.

\subsection{Caracterización físico-química}

Los resultados obtenidos en los análisis físico-químicos se muestran en la Tabla 5, donde se muestran las medias y desviación estándar de cada análisis realizado.

Tabla 4. Composición proximal de los tratamientos analizados

\begin{tabular}{lcccccc}
\hline \multicolumn{1}{c}{ Tratamiento } & $\begin{array}{c}\text { Proteína } \\
(\%)\end{array}$ & $\begin{array}{c}\text { Grasa } \\
(\%)\end{array}$ & $\begin{array}{c}\text { AGS } \\
(\%)\end{array}$ & $\begin{array}{c}\text { AGMI } \\
(\%)\end{array}$ & $\begin{array}{c}\text { AGPI } \\
(\%)\end{array}$ & $\begin{array}{c}\text { AGT } \\
(\%)\end{array}$ \\
\hline $\begin{array}{l}\text { Salchicha } 100 \% \\
\text { grasa dorsal de }\end{array}$ & $13.23 \pm 0.77^{\mathrm{a}}$ & $16.93 \pm 1.06^{\mathrm{a}}$ & $8.46 \pm 0.28^{\mathrm{a}}$ & $2.99 \pm 0.10^{\mathrm{a}}$ & $4.56 \pm 0.15^{\mathrm{a}}$ & 0 \\
cerdo & & & & & & \\
Salchicha 100\% \\
$\begin{array}{l}\text { aceite de } \\
\text { aguacate }\end{array}$
\end{tabular}




\subsubsection{Determinación de proteína}

Los promedios de los resultados obtenidos en la determinación de proteína agrupados por tratamientos se muestran en la Figura 7.

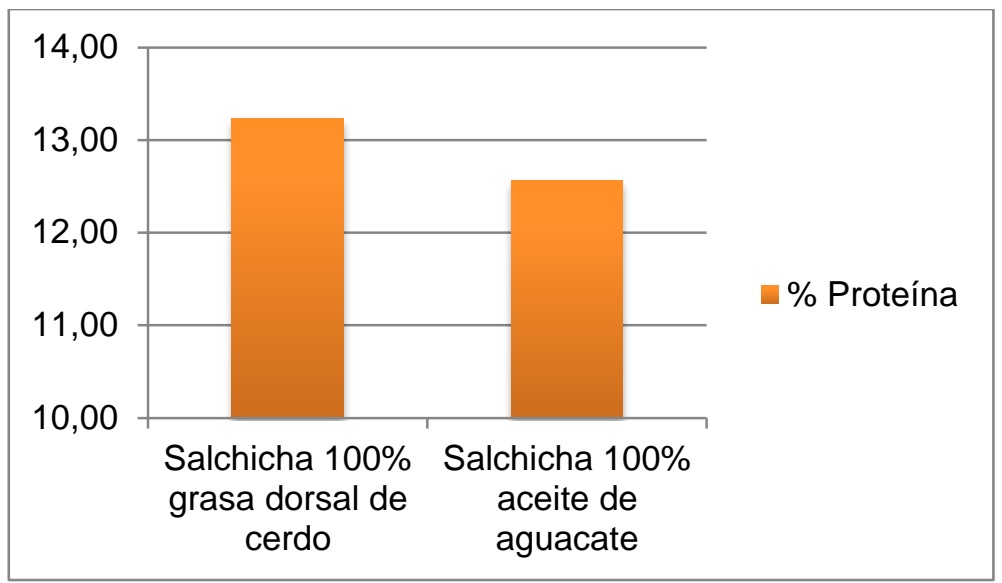

Figura 7. Contenido de proteína de los tratamientos analizados

En el tratamiento que contenía $100 \%$ de aceite de aguacate, se observa una reducción en el contenido de proteína en un $0.67 \%$ en relación al tratamiento con $100 \%$ de grasa dorsal en su formulación.

(Yildiz \& Serdaroglu 2012), reportan de igual manera que al remplazar la grasa dorsal de cerdo por aceite de avellana en un 60 - 90 \% en la elaboración de salchichas, existió una variación en el contenido de proteína de los tratamientos evaluados, sin embargo no representó una diferencia estadísticamente significativa.

La grasa dorsal de cerdo contiene entre 2.7 y 8.9 \% de proteína (FAO 2007, Mataix 2009). La proteína encontrada en el tejido adiposo proviene del tejido conectivo donde los animales bien alimentados almacenan grasa. Este tejido conectivo forma la fascia que se encuentra en la capa adiposa. La fascia es una membrana conjuntiva que limita el músculo de la grasa y de otros músculos formando grupos funcionales; proporciona una matriz de sostén además de permitir a la grasa adherirse extensamente a los músculos. Las proteínas que componen este tejido conectivo son el colágeno y elastina (Chaitow \& Walker2006, Grüner, et al. 2005).

\subsubsection{Determinación de grasa total}

Los promedios de los resultados obtenidos en la determinación de grasa total agrupados por tratamientos se muestran en la Figura 8.

El contenido de grasa total se redujo en $3.7 \%$ en el tratamiento que contenía $100 \%$ de aceite de aguacate en sustitución de la grasa de cerdo en su formulación. 


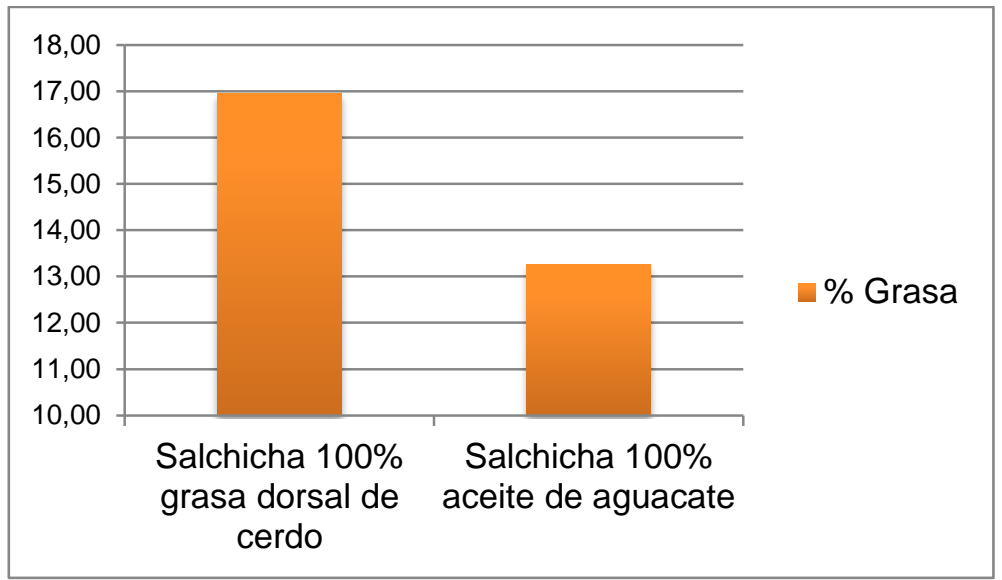

Figura 8. Contenido de proteína de los tratamientos analizados

Pascual et al. (2002), de igual manera indica una reducción en el contenido de grasa total en salchichas tipo Frankfurt con aceite de oliva en su formulación en comparación al contenido encontrado en el tratamiento control el cual contenía $100 \%$ de grasa dorsal.

(Yildiz \& Serdaroglu 2012), reportan resultados similares a los presentados en esta investigación, ya que al sustituir la grasa dorsal de cerdo únicamente con aceite de avellana, se alcanzó una reducción del 0.05 - $0.13 \%$ en el contenido de grasa total de los ensayos experimentales contrastando con el contenido del tratamiento testigo, al tener este porcentaje bajo de reducción de grasa total los autores declaran que no existieron diferencias significativas en el contenido de grasa total al utilizar aceite de avellana.

\subsubsection{Determinación de perfil lipídico de los tratamientos evaluados}

Los promedios de los resultados obtenidos en la determinación de perfil lipídico agrupados por tratamientos se muestran en la Figura 9.

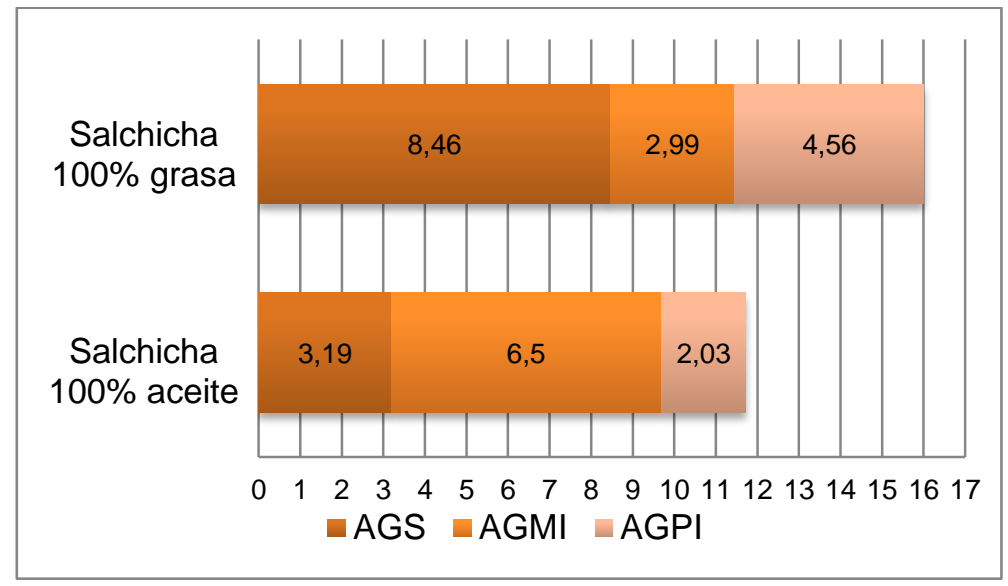

Figura 9. Perfil lipídico de los tratamientos evaluados

Existe una disminución en el contenido de AGS en el tratamiento con 100\% de aceite de aguacate, probablemente debido a que la grasa dorsal de cerdo contiene un $35.45 \%$ de AGS contra el $18.61 \%$ presente en el aceite de aguacate. 
Se determinó un aumento en el contenido de AGMI en el tratamiento con $100 \%$ de aceite de aguacate, posiblemente a causa del contenido de este tipo de ácidos grasos en el aceite de aguacate, el cual es de $68.37 \%$ contra un $41.95 \%$ presente en la grasa dorsal de cerdo.

Se estableció una disminución en el contenido de AGPI en la salchicha elaborada con aceite de aguacate en relación al tratamiento control con grasa de cerdo. Esta variación pudo haber sido ocasionada por el contenido de AGPI presente en el aceite de aguacate que es de $12.19 \%$, mientras que en la grasa de cerdo este contenido es mayor llegando a $20.71 \%$. El alto nivel de insaturación de la grasa de cerdo pudo deberse al uso de alimentos balanceados ricos en ácido linoleico como son el maíz o la soja en la dieta de los cerdos (Bañon et al. 2000).

(Yildiz \& Serdaroglu 2012), reportan resultados similares a los presentados en este estudio respecto al perfil lipídico de salchichas, los autores indican una disminución del contenido de AGS en la salchicha elaborada utilizado un $90 \%$ de aceite de avellana en sustitución de la grasa dorsal de cerdo. Adicionalmente muestran un aumento del contenido de AGMI en relación a su tratamiento control.

En los dos tratamientos evaluados se obtuvo $0 \%$ ácidos grasos trans, lo cual indica que no existió hidrogenación biológica en la grasa de cerdo como consecuencia de la degradación bacteriana de ácidos grasos; de igual manera no existió hidrogenación debida al calentamiento en el proceso de cocción en el aceite de aguacate (Panisello 2004, Ruiz 2009, Serna 2010).

\subsection{Caracterización microbiológica}

Los promedios de los resultados obtenidos en la determinación de perfil lipídico agrupados por tratamientos se muestran en la Tabla 6.

Se identificó la presencia de Aerobios mesófilos y Staphilococcus aureus en los tratamientos analizados, sin embargo se logró notar una menor carga microbiana en el tratamiento con $100 \%$ de aceite de aguacate en su formulación. (Pascual \& Calderón 2000), indican que el crecimiento bacteriano en aceites y grasas, a excepción de margarinas, es muy limitado y en la mayoría de casos llega a ser nulo, ya que las baterías no pueden multiplicarse en un producto si este no contiene agua, razón por la cual los aceites generalmente no suponen un problema de carácter sanitario.

La norma CODEX STAN 19-1981 para grasas y aceites comestibles no regulados por normas individuales, establece un contenido máximo de humedad del $0.2 \%$, mientras que la norma NMX F-052 para aceite de aguacate, establece en sus especificaciones un contenido 
de humedad máximo de $0.5 \%$ para aceite de aguacate y un $0.1 \%$ de humedad para aceite comestible puro de aguacate IMNC. (2007).

Tabla 5. Resultados de análisis microbiológicos y requisitos según norma INEN 1338:12 para productos cárnicos cocidos

\begin{tabular}{|c|c|c|c|c|}
\hline \multirow[b]{2}{*}{ Requisito } & \multirow[t]{2}{*}{$\begin{array}{c}\text { Salchicha } \\
100 \% \text { grasa } \\
\text { dorsal de } \\
\text { cerdo }\end{array}$} & \multirow[t]{2}{*}{$\begin{array}{c}\text { Salchicha } \\
100 \% \text { aceite } \\
\text { de } \\
\text { aguacate }\end{array}$} & \multicolumn{2}{|c|}{$\begin{array}{c}\text { Requisitos norma INEN } \\
1338: 12\end{array}$} \\
\hline & & & $\begin{array}{l}\text { Nivel de } \\
\text { aceptación }\end{array}$ & $\begin{array}{l}\text { Nivel de } \\
\text { rechazo }\end{array}$ \\
\hline Aerobios mesófilos ufc $/ \mathrm{g}^{*}$ & $5.6 \times 10^{4}$ & $4.1 \times 10^{4}$ & $5.0 \times 10^{5}$ & $1.0 \times 10^{7}$ \\
\hline Escherichia coli ufc/g* & Ausencia & Ausencia & $<10$ & - \\
\hline Staphilococcus aureus ufc $/ \mathrm{g}^{*}$ & $1.8 \times 10^{2}$ & $1.0 \times 10^{2}$ & $1.0 \times 10^{3}$ & $1.0 \times 10^{4}$ \\
\hline Salmonella $125 \mathrm{~g}$ & Ausencia & Ausencia & Ausencia & - \\
\hline
\end{tabular}

${ }^{1}$ Especies cero tipificadas como peligrosas para humanos

* Requisitos para determinar término de vida útil

${ }^{\star *}$ Requisito para determinar inocuidad del producto

(FAO 2007), establece un contenido de humedad del $7.7 \%$ para la grasa dorsal de cerdo al ser un producto fresco. La grasa dorsal de cerdo al contener mayor porcentaje de humedad y al ser un producto fresco es más susceptible a ataques microbianos, mientras que el aceite de aguacate al contener un porcentaje de humedad relativamente bajo y ser un producto procesado, presentará una carga microbiana muy baja.

\section{Conclusiones y Recomendaciones}

La grasa dorsal de cerdo presentó un nivel de instauración bastante elevado debido a su alto porcentaje de ácido linoleico; por su parte el alto contenido de ácidos grasos insaturados encontrados en el aceite de aguacate permitió vislumbrar las características nutraceúticas del mismo.

Se consiguió reemplazar en su totalidad la grasa animal presente en la formulación, logrando además conservar las características sensoriales y tecnológicas propias de este tipo de producto cárnico.

Se determinó la aceptabilidad del producto obtenido a través de un análisis sensorial; el tratamiento con $100 \%$ de aceite de aguacate en sustitución de la grasa dorsal de cerdo alcanzó una puntuación de 5.82 / 9, logrando de esta manera obtener el mejor resultado de aceptabilidad a pesar de no haber sido altamente puntuado en sus atributos de sabor y textura por la ausencia de grasa dorsal en su formulación.

Se determinó que el tratamiento patrón contenía mayor porcentaje de proteína, sin embargo ambos tratamientos reportaron más del $12 \%$ de proteína, por lo cual de acuerdo a la norma INEN 1338:12, se los considera como salchichas tipo I. 
La adición de aceite de aguacate alteró la composición de ácidos grasos de las salchichas elaboradas, ya que se encontraron diferencias significativas para los tratamientos evaluados respecto a la composición de ácidos grasos saturados e insaturados.

Se identificó la presencia de Aerobios mesófilos y Staphilococcus aureus en los tratamientos analizados, no obstante los resultados reportados en son inferiores a los valores dictados por la norma INEN 1338:12, por lo cual de acuerdo a la legislación normativa ecuatoriana los embutidos elaborados cumplen con los requisitos de seguridad alimentaria.

Se cuantificó una carga microbiana menor en el tratamiento con $100 \%$ de aceite de aguacate en su formulación, debido a que la grasa dorsal de cerdo al contener mayor porcentaje de humedad y al ser un producto fresco fue más susceptible a ataques microbianos, mientras que el aceite de aguacate al contener un porcentaje de humedad relativamente bajo y ser un producto procesado, presentó una carga microbiana muy baja.

Los resultados encontrados permiten el planteamiento de nuevos temas de investigación con relación a la sustitución de grasa dorsal de cerdo utilizando aceite de aguacate como el estudio de la vida útil del producto a nivel microbiológico, ya que el aceite de aguacate al no ser susceptible a ataques microbianos podría influir en el crecimiento de bacterias en el embutido.

\section{Bibliografía}

Acosta, M. (2011). Evaluación y escalamiento del proceso de extracción de aceite de aguacate utilizando tratamiento enzimático. Tesis de Pregrado de Ingeniería Química y Ambiental. Universidad Nacional de Colombia. Bogotá, Colombia.

AOAC. (2012). Official Methods of Analisis (19th Ed.). Association of Official Analytical Chemists. Gaithersburg, USA.

Banda, D. (2010). El Efecto de la sustitución de grasa animal (cerdo) por grasa vegetal (Danfat FRI - 1333) en la formulación y elaboración de salchichas Frankfurt. Tesis de Pregrado de Ingeniería de Alimentos. Universidad Técnica de Ambato. Ambato, Ecuador.

Bañón, S., Granados M., Cayuela J., Gil, M., Costa E., \& Garrido M. (2000). Calidad de la Grasa Obtenida a Partir de Cerdos Magros, Anales de Veterinaria de Murcia, 16, 7788.

Chaitow, L., \& Walker, J. (2006). Aplicación clínica de las técnicas neuromusculares. (Vol. II). Badalona: Editorial Paidotribo. 
Diario La Hora. (2013). El "semáforo" alimenticio tiende al amarillo. Recuperado el 01 de junio de 2014, de La Hora: http://www.lahora.com.ec.

FAO. (2007). Meat processing technology for small- to medium- scale producers. Bangkok: FAO.

García, H., Palo, P., \& Salas, J. (2008). Sustitución De Grasa Animal Por Grasa Vegetal En La Elaboración De Salchicha Frankfurt, Revista Salud Pública y Nutrición, 6(6), 42-45.

Grüner, H., Metz, R., \& Gíl, A. (2005). Procesos de cocina. Madrid: Akal S.A.

IMNC. (2007). Aceites y grasas. Aceite de aguacate. Especificaciones. Instituto Mexicano de Normalización y Certificación, F 052.

INEC. (2011). Registro de Defunciones 2011. Recuperado el 25 de Mayo de 2013, de INEC: http://www.inec.gob.ec.

INEN. (2012). Carne y productos cárnicos. Productos cárnicos crudos, productos cárnicos curados-madurados y productos cárnicos precocidos-cocidos. Requisitos. Instituto Nacional Ecuatoriano de Normalización, 1338.

Mataix, J. (2009). Tabla de composición de alimentos (5ta Ed.). Granada: EUG.

Obenland, D., Collin, s., Sievert, J., Negm, F., \& Arpaia, M. (2012). Influence of maturity and ripening on aroma volatiles and flavor in 'Hass' avocado. Postharvest Biology and Technology, 71, 41-50.

Olaeta, J. (2003). Industrialización del aguacate: estado actual y perspectivas futuras. Proceedings V World Avocado Congress, 749-754.

Panisello, J. (2004). Las 100 preguntas que siempre quiso hacer: mitos y verdades en torno a la alimentación (Vol. I). Barcelona: Glosa.

Pascual, CH., Ordoñez, S., \& Olivares, G. (2002). Elaboración de Salchicha tipo Frankfurt con aceite virgen de oliva, Revista Anales Científicos, 195-219.

Pascual, M., \& Calderón, V. (2000). Microbiología Alimentaria: Metodología Analítica para Alimentos y Bebidas (2da ed.). Madrid: Díaz de Santos.

Ruiz, V. (2010). Acidos grasos trans. Recomendaciones para reducir su consumo, Revista cubana de alimentación y nutrición, 19(2), 364-369.

Selgas, M., Cáceres, E., García, M. (2005). Long-chain soluble dietary fibre as functional ingredient in cooked meat sausages, Food Science and Technology International, 11(1), 1-7. 
Serna, I. (2010). Guía de Nutrición y Alimentación Saludable: Los Macronutrientes: Hidratos de Carbono, Grasas y Proteínas (4ta ed.). Valencia: Concejalía de Sanidad del Ayuntamiento de Valencia.

Yildiz-Turp, G. \& Serdaroglu, M. (2012). Partial Substitution of beel Fat with Hazelnut Oil Type Sausages: Effects on Chemical, Physical and Sensorial Quality, Journal of Food Technology, 10(2), 32-38. 